\title{
Zur Wirkung der Worte. Sprachliche Bewertungsmittel als Indikatoren der Wortkraft in der emotionsbezogenen NS-Sprache
}

\author{
The effect of the words. Linguistic assessment means as indicators
} of word power in the emotion-related language of National Socialism

\author{
Katarzyna KUKOWICZ-ŻARSKA ${ }^{1}$ \\ Ateneum-Szkoła Wyższa w Gdańsku
}

\begin{abstract}
The subject of this publication is to investigate the power of words in a text and language in the context of evaluation. The author focuses on emphasizing the role of evaluation in the text and the relationship between the evaluation process and accompanying and/or resultant emotions. The evaluative language measures applied in the text under analysis are addressed on the basis of the strongly emotional German language of the National Socialism era. The paper pays particular attention to the influence of emotions, emotionally influenced language and the socio-cultural context on the recipient and his/her mental responses as well as his/her verbal and physical actions. Actions implemented through the text are perceived here through the prism of universal values, as well as through the prism of specific evaluative methods relevant to the applied evaluative language means.
\end{abstract}

Keywords: text linguistics, evaluation, axiology, valuation, sociolinguistics, German language

\section{Zusammenfassung}

Das Thema der Publikation ist es, die Macht der Worte im Text und in der Sprache im Kontext der Bewertung zu schildern und zu kommentieren. Die Autorin konzentrierte sich darauf, die Rolle der Bewertung im Text zu betonen sowie die Zusammenhänge zwischen dem Bewertungsprozess und Emotionen, die ihn begleiten oder dessen Ergebnis sie sind, aufzuzeigen.

1 https://orcid.org/0000-0002-6652-9068. 
Die im Text vorgestellten Sprachbewertungsmaßnahmen werden auf der Grundlage der stark emotional geprägten Sprache der NS-Zeit in Deutschland diskutiert. Eine besondere Rolle in der Publikation spielt der Einfluss von Emotionen, emotional geprägter Sprache sowie soziokulturellem Kontext auf den Empfänger und seine mentalen Reaktionen sowie seine verbalen und physischen Handlungen. Die durch den Text implementierten Handlungen werden hier durch das Prisma der Werte in einem universellen Kontext verstanden sowie durch das Prisma spezifischer Bewertungsmethoden wahrgenommen, die unter Verwendung der beschriebenen sprachlichen Bewertungsmittel implementiert werden.

Schlüsselwörter: Textlinguistik, Evaluation, Axiologie, Bewertung, Soziolinguistik, deutsche Sprache

\section{Problemdarstellung}

„Diktaturen sind nie sprachlos“, konstatiert Schlosser (2013, S. 9). Die NSHerrschaft war in dieser Hinsicht keine Ausnahme - im Gegenteil: in der Ära des Nationalsozialismus spielte die Sprache die höchstrangige Rolle in der Gesellschaft. Die Sprache wurde in dieser Diktatur „neben und nicht selten vor physischer Gewalt zum unverzichtbaren Instrument (...), zunächst die Macht zu erringen und diese dann so lange wie irgend möglich zu erhalten" (ebd.). Die Wortkraft war also in dieser emotionsbezogenen und instrumentalisierten Sprache nicht zu überschätzen - erstens wurde die Macht durch entsprechende Worte signifikant unterstützt, zweitens gewährleisteten entsprechende Worte, dass die errungene Macht erhalten bleibt, letztendlich resultierte diese Wortkraft ,aus der Angst der Mächtigen vor dem unkontrollierten freien Wort, der oft einzigen ,Waffe' einer Opposition“ (ebd.), was besondere Schritte erforderte. Die vorliegende Arbeit setzt sich deswegen zum Ziel, Phänomene der NS-Sprache in Deutschland auf der lexikalischen sowie semantischen Ebene wissenschaftlich zu erörtern, wobei im Vordergrund die Fragen der Bewertung und der Weltwahrnehmung durch die auf Emotionen bezogene Sprache gestellt werden sollen². Als These soll angenommen werden, dass eine stark emotionsbezogene Sprache, wie es eben die NS-Sprache war, einen besonderen Fall der Bewertung bilden und als solche spezifisch evolviert werden kann, und dass sie imstande ist, einen besonderen Einfluss auf ihren Rezipienten auszuüben. Als Indikatoren der

\footnotetext{
2 Einige Abschnitte dieses Beitrages sind aus projektspezifischen sowie sprachbezogenen Gründen mit Kukowicz-Zarska 2018 (auf Polnisch) teilweise konvergent. Der polnische Titel der vorliegenden Publikation lautet: „O wartościowaniu $i$ prawdzie $w$ języku i tekście. Język narodowego socjalizmu w perspektywie językoznawczej i socjokulturowej”.
} 
Wortkraft in der emotionsbezogenen Sprache des Nationalsozialismus fungieren die im Text geschilderten sprachlichen Bewertungsmittel, die die Wirkung der Worte prägnant schildern.

Die im weiteren Teil des Artikels geschilderten Auffassungen und Erwägungen sollten zusammen mit meiner früheren Publikation in der polnischen Sprache (Kukowicz-Żarska, 2018) als Ganzes betrachtet werden, denn der vorliegende Beitrag selbst versteht sich als eine Fortsetzung und Erweiterung der früheren wissenschaftlichen Untersuchungen über die Bewertung und Wahrheit in der Sprache aus sprachwissenschaftlicher sowie soziokultureller Perspektive ${ }^{3}$. Der Artikel ist ein Versuch, das Problem der Essenz von Bewertung und Wahrheit in der Sprache und im Text wissenschaftlich zu untersuchen. Der Gegenstand dieser Veröffentlichung sind also sprachliche Bewertungsmittel im Text, die auf der Grundlage der deutschen Sprache in der Zeit des Nationalsozialismus diskutiert werden. Der Text und seine Rolle werden hier durch das Prisma der Werte in einem universellen Kontext sowie - und vielleicht vor allem - durch das Prisma der darin dargestellten sprachlichen Bewertungsmittel wahrgenommen. Es sollte nicht wundern, dass die Sprache des Nationalsozialismus sowohl in der sprachlichen als auch soziokulturellen Perspektive erörtert wird, da wir in der Regel ausgewählte Elemente der Realität beurteilen, die mit Hilfe der Sprache erstellt wurden, und zwar so, dass diese Beurteilungen nicht nur unsere Einstellung $\mathrm{zu}$ diesen Elementen widerspiegeln, sondern auch den Empfänger einer Sprachnachricht explizit bzw. implizit zu der vom Emittenten festgelegten und gewünschten mentalen bzw. physischen Reaktion zwingen sollten.

3 Den Anlass zu all den geschilderten Erwägungen hatte die Lektüre der Publikation von Manfred Pechau, einem Germanisten der Epoche des Nationalsozialismus in Deutschland, gegeben (Kukowicz-Żarska, 2018, S. 62f.). Sein damals als wissenschaftlich fungierter Artikel unter dem Titel „Nationalsozialismus und deutsche Sprache” wurde 1937 in „Nationalsozialistischen Monatsheften” veröffentlicht. Die erwähnte Publikation zur Sprache des Nationalsozialismus bildet eine Zusammenstellung der wichtigsten Thesen, die Pechau auch in seiner gleichnamigen Dissertation beinhaltet hat, auf deren Grundlage er 1935 an der ErnstMoritz-Arndt-Universität in Greifswald promoviert wurde. 


\section{Die Sprache des Nationalsozialismus im Lichte der wissenschaftlichen Debatte}

Bevor wir uns mit der Analyse der Sprache des Nationalsozialismus und all den damit verbundenen sprachlichen sowie soziokulturellen Aspekten befassen werden, sollte man in diesem Zusammenhang eine grundlegende Frage stellen: Existiert das Phänomen der "Sprache des Nationalsozialismus" überhaupt, oder handelt es sich nur um eine Erscheinung, die für die Zwecke der Debatte und Gesellschaftskritik geschaffen wird?

Senya Müller (1994, S. 25) versucht diese Frage ebenfalls zu erörtern, indem sie eine chronologische Übersicht über die Auffassungen verschiedener Wissenschaftler zu diesem Thema schildert. Für eine adäquate Beschreibung der Situation der NS-Sprache in Deutschland zwischen 1933 und 1945 schlagen also Forscher eine Reihe abwechslungsreicher Konzepte vor: $L_{T I}^{4}$ (Klemperer, 1947), Sprache des Dritten Reiches, Nazi-Deutsch (Paechter, 1944), Vokabular des Nationalsozialismus (Berning, 1964), Wortschatz eines Unmenschen (Sternberg, Storz und Süskind, 1967), Sprachwandel im Dritten Reich (SeidelSlotty, 1961), Sprache unterm Hakenkreuz (Schlosser, 2013). Obwohl also viele Wissenschaftler die Idee der Existenz des Konstrukts "Sprache des Nationalsozialismus" (dt. auch: NS-Sprache) stark abzulehnen scheinen, indem sie argumentieren, dass viele Merkmale dieser auch vor 1933 im Deutschen erkennbar waren, und sie daher nicht als distinktive Eigenschaften der Sprache der Nationalsozialismus anerkannt werden sollten, kommt dieser Begriff in Studien zur deutschen Sprache dieser Zeit weitgehend ziemlich konsequent vor. Es ist eine unbestrittene Tatsache, dass dieser in der NS-Zeit dominierende Sprachgebrauch zur offiziellen Staatssprache transformiert werden sollte, „indem vorhandene Sprachmuster mit ideologisch und politisch gewünschten Bedeutungen besetzt, bestimmte Elemente von Wortschatz und Satzbau in ihrem Gebrauch monopolisiert und andere, die abweichende Meinungen hätten fördern können, tabuisiert wurden." (Schlosser, 2013, S. 10). Man kann

\footnotetext{
${ }^{4}$ LTI - Lingua Tertii Imperii also Sprache des Dritten Reiches - eine Abkürzung, abgeleitet vom Buchtitel von Victor Klemperer: LTI. Notizbuch eines Philologen, (1947, Berlin: Aufbau-Verlag), also einer semantischen Studie zur Sprache des damaligen Deutschlands. Es ist ein hinterhältiges Wortspiel, das ironisch auf die deutschen Akronyme verweist, die von Nazi-Behörden häufig verwendet wurden: NSDAP, KDF, HJ. BDM etc.

${ }^{5}$ So lautet auch der Titel seines Buches (Schlosser, 2013), der auch nach dem Autor zum Ausdruck bringen sollte, dass zwischen 1933 und 1945 das allgegenwärtige Symbol der NSGewaltherrschaft über jeglichem Sprechen und Schreiben wie über allem, was in Deutschland geschah, wie ein Damoklesschwert hing." (ebd., 10).
} 
also wie Victor Klemperer (1947) von der Sprache des Dritten Reiches - Lingua Tertii Imperii - sprechen, weil die damalige Sprache, oder die Sprache im Dritten Reich, wie es die neueren Forschungen eher betonen möchten, „insgesamt so sehr infiziert war, dass sich (...) selbst der Widerstand den Wirkungen der offiziellen Sprache, des sogenannten Offizialidioms, nicht ganz entziehen konnte“ (ebd.).

Müller (1994, S. 11) bemerkt anschließend, dass die Redeweise und Ausdrucksform des "Dritten Reichs", die in den Äußerungen der neuesten Zeit wieder auferstanden sind, schon nach 1945 eine heftige Diskussion ausgelöst haben. Das Verhältnis zum Thema der Sprache der NS-Ära hat sich jedoch zwischenzeitlich verändert:

Zunächst versteift man sich darauf, das inkriminierte Wortgut in Listen zu sammeln, die "Sprache des NS" als eine moralisch verwerfliche darzustellen. Wörter wie Volksgemeinschaft, Rassenschande, aber auch einfachere Vokabeln wie betreuen oder durchführen werden geächtet. Später wird dazu übergegangen, eine wissenschaftlichere, auf weniger moralischen Prämissen aufgebaute Analyse zu betreiben. Man kommt überein, die "braune" Diktion als eine Erscheinung im historischen und gesamtsprachlichen Zusammenhang zu betrachten, nicht als eine Sprache, die nach 1933 entstanden und nach dem Kriege wieder verschwunden ist. Vokabeln wie betreuen oder durchführen werden geächtet. (Müller, ebd.).

Deshalb werde ich auch im weiteren Teil der vorliegenden Publikation das Konzept der Sprache des Nationalsozialismus als eine gewisse Verallgemeinerung verwenden und dies für die Beschreibung der nationalsozialistisch geprägten Sprache in den Jahren 1933-1945 in Deutschland anerkennen. Dieses Phänomen wird anschließend in seiner charakteristischsten Schicht, d.h. auf der lexikalischen Ebene betrachtet, wobei bei der textlinguistischen Analyse gleichzeitig auch lexikalische Semantik und stilistische Prägung berücksichtigt werden sollen sowie eine deutliche Bereicherung mit soziokulturellen Aspekten der betroffenen Epoche. Damit also erhalten analysierte Phrasen einen bestimmten Status, der über die Sprache als solche hinausreicht, der in diesem Fall die endgültigen Obertöne bestimmt und die Art der Auswirkung auf den damaligen Rezipienten äußert, da er gleichzeitig das Damalige abbildet und bewertet. 


\section{Die Rolle der Sprache und ihrer Kraft für die NS-Diktatur}

Die erste Studie über die Sprache des Nationalsozialismus soll die bereits oben genannte Dissertation Manfred Pechaus ${ }^{6}$ „Nationalsozialismus und deutsche Sprache" (und die danach auf ihrer Basis entstandene Publikation unter demselben Titel) sein (Kämper-Jensen, 1993, S. 160). In seinen Analysen will er erkannt haben, dass der NS-Wortschatz hauptsächlich aus alten und bekannten Wörtern bestehe, die jedoch mit einem neuen semantischen Gehalt versorgt werden sollten. Pechau geht sogar weiter und stellt pathetisch hochtrabend fest: „Das Hauptgewicht der nationalsozialistischen Sprachbeeinflussung liegt auf der neuen Sinngebung oft alter, bekannter Worte" (Pechau, 1935, S. 11). Kämper-Jensen nennt die in der Publikation dargestellten Erwägungen als „eine systematische Betrachtung des nationalsozialistischen Wortschatzes"7, in der „Ausdrücke der Parteiorganisation“, „Kampfsprachformen“ und „Sprachformen aus dem Kampf gegen das Judentum“ untersucht werden sollten. Im zweiten Teil dagegen werden wortgeschichtliche Betrachtungen präsentiert, wo Pechau als aktiver Nationalsozialist „ganz im Sinne Hitlers auf die Bedeutung des Propagandastils, d. h. eine allein auf Wirkung bedachte sprachliche Darbietung, die er als „Stil der Zweckmäßigkeit" bezeichnet," verweise (Kämper-Jensen, 1993, S. 160).

\footnotetext{
${ }^{6}$ Nach Simon (2003, S. 4) sollte Pechau das Thema „Nationalsozialismus und deutsche Sprache“ schon 1932 von seinem Doktorvater Wolfgang Stammler für eine Seminararbeit gestellt werden, erst danach wurde es als Titel einer Dissertation angeboten. Diese wurde am 10. August 34 als Leistung für eine mit "gut“ bestandene Doktorprüfung von der Preußischen Ernst-Moritz-ArndtUniversität zu Greifswald anerkannt. 1935 erschien sie im Druck, und danach erschien ihre Kurzfassung angereichert durch neue Beispiele aus unbekannten Gründen im Dezember 1937 in den „Nationalsozialistischen Monatsheften“ (ebd.).

${ }^{7}$ Lerchenmüller und Simon (2009, S. 93) äußerten sich präzise und gleichzeitig provokativ zum wissenschaftlichen Wert der Dissertation von Manfred Pechau indem sie auf höchst wertende Art und Weise festgestellt haben, dass es sich dabei nun um „einen am Sonntag Nachmittag hingeschluderten Schulaufsatz" handeln sollte, der schnell und unzuverlässig zusammengestellt wurde. Zusätzliche Informationen liefert der zweite Teil des zuvor zitierten Titels von Simon (2003): „NS-Sprache aus der Innensicht. Der Linguist Pechau und der Massenmord in den Sümpfen bei Loknja.“ Die Gegenüberstellung von Pechau mit einem Massenmord impliziert seine Teilnahme an diesem Ereignis und bewertet daher nicht nur die Handlung selbst, sondern auch die erwähnte Person mit einer eindeutig negativen Konnotation. In seiner Veröffentlichung beschrieb Simon diese "wissenschaftliche" Sphäre von Pechau's Aktivität, widmete aber auch seiner nichtwissenschaftlichen Tätigkeit viel Aufmerksamkeit und nannte Pechau dabei einfach Kriegsverbrecher. All diese Bemühungen schmälern die Bedeutung des zitierten Autors und verringern somit die Rolle seiner eventuellen wissenschaftlichen Leistungen.
} 
Im Kontext der Wort- und Sprachforschung, der wissenschaftlichen Arbeit also, die hohen Anforderungen von „politischer Ausrichtung des deutschen Menschen“ sowie von "politischer Ausrichtung des wissenschaftlichen Arbeiters" unterliegen sollte (Pechau, 1937, 2 [1958]), scheint es sinnvoll zu sein, die Einstellung des Autors selbst der Sprache gegenüber zu schildern. Pechau (1935) versteht also die Sprache folgendermaßen:

Die Sprache ist der Ausdruck des Wollens und Denkens der Menschen und bleibt stets Verkörperung der Seele. Sie gibt wieder, was das Innere des Menschen bewegt und lässt das Denken eines Volkes erkennen. Und wie das Denken der Völker sich wandelt im Laufe der Geschichte, wie es den Gesetzen der Entwicklung zu folgen hat, so ist auch die Sprache einer fortlaufenden Entwicklung unterworfen und getreulich ein Spiegelbild der Zeit, die sie schuf. (ebd., S. 9)

Diese oben zitierte pathetische Auffassung präsentiert eindeutig die sprachtheoretische Grundposition der weiteren theoretischen Überlegungen. Pechau argumentiert für eine relevante und sogar führende Rolle der Sprache für das Funktionieren der gesamten Nation (des Volkes) und befasst sich in diesem Zusammenhang besonders ausführlich mit dem Thema "Wort- und Sprachforschung" (1937, S. 2 [1058]). Diese Untersuchungen versteht er jedoch nicht als „Privatspielerei“, sondern viel breiter, als eine explizite, kategorische Volksidee. Die deklarativen Feststellungen, bei denen die Modalität mit Hilfe von müssen-Sätzen explizit geäußert wird, haben eine bewertende Funktion, indem sie die Tatsache betonen, dass die Arbeit eines Sprachwissenschaftlers nicht die weniger wesentlichen Einzelbestrebungen verschiedener Personen, sondern die Bedürfnisse der gesamten Nation verfolgen sollte: „Dem Sprachforscher aber ist es klar, daß (...) seine Arbeit den Marschtritt des Volkes erklingen lassen muß, den die Sprache so getreulich wiedergibt" (ebd.). Betont werden muss, dass in seinem Text immer wiederkehrend im Kontext der Nation der Begriff Volk vorkommt (Kukowicz-Żarska, 2018, S. 71f.). 


\section{Bewertung als Realisation der emotionalen Prägung der Worte}

Emotionen begleiten jeden Menschen in jeder beliebigen Phase seines Lebens und ermöglichen, verschiedene verbale, mentale sowie physische Reaktionen auszulösen. Diese Anmerkung kann auch nachhaltig und zielgemäß dazu beitragen, dass jede Emotion als „Sonderfall des Bewertens“ definiert werden kann (Sandig, 2007, S. 167). Aus einer anderen Perspektive gesehen - die Bewertung ist als ein inhärentes Merkmal von Emotionen anzusehen (Jahr, 2000, S. 66). Dies bedeutet, dass sobald in der Kommunikation Emotionen in Frage kommen, dabei auch gleichzeitig Bewertungen kommuniziert werden. Fries (2007, S. 295) erklärt das Phänomen der umfassenden Kommunizierbarkeit von Gefühlen dadurch, dass man sie in interaktiven Prozessen vermitteln und thematisieren kann (also man kann über sie reden), sowie dass man sie inszenieren kann (also ihr Ausdruck kann zum Zweck zielorientierten Handelns eingesetzt werden). Gefühle sind also kommunizierbar, „sofern für sie konventionalisierte Zuordnungen von Zeichenformen und Zeichenbedeutungen konstatiert werden können“ (ebd.). Die für die Linguistik relevante generalisierbare Funktion von Gefühlen besteht nach Fries $(2004$, S. 7) darin, emotionale Bewertungen über sprachliche Äußerungen sowie ihre Komponenten zu liefern, hierdurch in Entscheidungsprozesse einzugreifen, sowie „die Planung, die Zielsetzung, den Verlauf und das Ergebnis von Handlungen zu beeinflussen"8.

Menschen unterliegen also den unterschiedlichsten Emotionen, und jede einzelne Emotion evoziert eine entsprechende emotionale Einstellung, die als Bewertung vermittelt werden kann. Diese emotionale Bewertung soll, mit Hilfe eines zu diesem Zweck hergestellten Textes, geäußert werden, um eine vorgeplante, gewünschte Reaktion/Handlung bei dem Rezipienten hervorzurufen. Emotionales Bewerten regt so die Textproduktion an, und es

$8 \mathrm{Um}$ das Verhältnis zwischen Handlungen, besonders zwischen Äußerungsbedeutungen und emotionalen Bewertungen zu erklären, verwendet Fries (2004, S. 7; 2007, S. 293f.) Begriffe: emotionale Einstellung und emotionale Szene - Gefühle beim Menschen sollen ,auf der Basis seiner semiotischen Kompetenzen im Ausdruck emotionaler Einstellungen sowie emotionaler Szene“ resultieren. Emotionale Einstellungen seien „Komponenten einer jeden sprachlichen Äußerungsbedeutung“, emotionale Szenen dagegen „signifikant relevante (komplexe) Konzepte für die Explikation der Elemente des Gefühlswortbestandes von Sprachen und von einschlägigen Metaphern und Metonymien“. Sie sollen eine Einwirkung auf die Prozesse der Planung, Zielsetzung, Realisierung sowie auf das Ergebnis von Handlungen haben, Entscheidungsprozesse mitbestimmen, und menschliche Fähigkeit zum Erinnern der Tätigkeiten, Erlebnisse, Urteile und Wünsche unterstützen. 
wird durch unterschiedliche Äußerungs- und Kommunikationsmittel unterstützt, die dem Textproduzenten helfen bzw. ermöglichen, sein gewünschtes Ziel zu realisieren. Die Textrezeption, die durch die Bewertung geprägt ist, fungiert als Ergebnis des ganzen Produktionsprozesses und soll eine gewünschte Handlung (die sowohl als mentale als auch physische bzw. sprachliche Reaktion verstanden werden kann) provozieren. Diese gegenseitigen Zusammenhänge können schematisch folgendermaßen dargestellt werden:

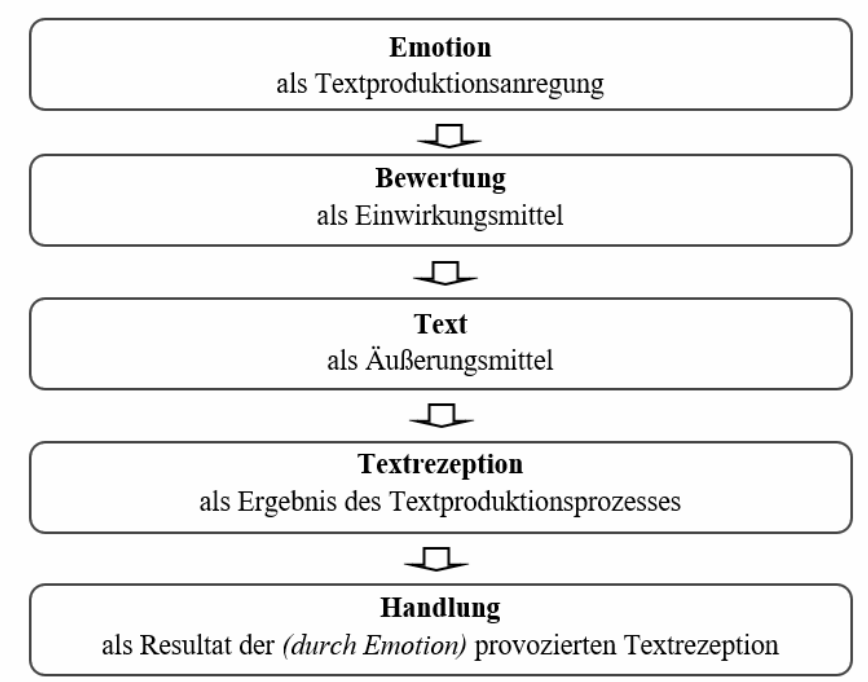

Man versucht dabei, die emotionale Bedeutung sprachlicher Äußerung insbesondere nach der Intensivität von Affekten, der positiven bzw. negativen Bewertung und der emotionalen Nähe von Sachverhalten zu differenzieren. Entscheidend sollte immer der Kontext sein, in dem ein Wort auftritt, weil er die Art und die Intensivität einer hervorgerufenen emotionalen Reaktion beeinflusst (Jahr, 2000, S. 64). Hier taucht aber eine prinzipielle Schwierigkeit auf, klare Grenzen zwischen emotionsgeprägten und emotionsfreien Wörtern zu bestimmen, da sogar „gefühlsmäßig indifferente Wörter je nach Kontext emotionale Reaktionen auslösen können“ (ebd.). 


\section{Bewertung im Kontext des Nationalsozialismus und seiner Sprache}

Die Bewertung erfolgt im Text mit Hilfe von sprachlichen Bewertungsmitteln und kann auf verschiedenen Ebenen erörtert werden. Wie jedoch Puzynina (1992) bemerkt: „Das Grundausdrucksmittel der Bewertung sind Worte“ (ebd., S. 118). Wenn also eine Emotion in der Interaktion kommuniziert wird, ist das gleichbedeutend mit der Kommunikation einer bewertenden Stellungnahme, oder allgemeiner ausgedrückt, einer Bewertung (Fiehler, 2001, S. 1429). Durch die sprachlich - durch Ausdrucksphänomene sowie Benennung in der gesprochenen Sprache und lexikalische Mittel in der geschriebenen Sprache unterstützte Realisation von den oben genannten Emotionen wird an den Kommunikationspartner eine spezifische negative bzw. positive Bewertung übermittelt.

Die uns interessierende Frage, wie Wörter eigentlich auf uns wirken, stellt auch Claudia Schmidt (2002, S. 64). Wie wirken Wörter? Dabei schildert die Autorin ein lexikalisches Konzept und erklärt es aus der Sicht der Psycholinguistik als eng „mit dem so genannten enzyklopädischen Wissen, unserem Alltagswissen“ verbunden (ebd.). Auf dieser Basis ist es möglich zu veranschaulichen, wie unser Wissen aktiviert wird, damit wir bestimmte Wörter bzw. Wortverbindungen rezipieren.

Da Schmidt (2002, S. 64) für die Veranschaulichung ihrer eigenen Erörterungen ein signifikantes Beispiel gewählt hat, das auch für unsere weitere Erörterungen vielsagend und von besonderer Bedeutung ist, scheint es an dieser Stelle angebracht $\mathrm{zu}$ sein, ihre Überlegungen näher zu thematisieren. Bei dem Beispiel geht es hier um die lexikalische Einheit Führer, die abhängig von bestimmten grammatischen und semantischen Informationen, ihrer eigenen lexikalischen Kraft oder vom (Satz)Kontext das Konzept aktiviert:

1. (der) Führer

2. der Führerschein

3. An der Expedition nahmen auch zwei einheimische Führer teil.

Das Wort Führer ohne Artikel wirkt auf den Rezipienten mit der Aktivation jemand, der eine Gruppe leitet, dieselbe Einheit mit dem bestimmten Artikel ruft jedoch die eindeutig negativ wertende Konzeptualisierung Hitler hervorruft. Dieses Lexem löst konzeptuelles Denken aus und unterstützt kognitive Prozesse, bei denen auch Emotionen einen wesentlichen kognitiven Anteil haben. Das Kompositum Führerschein aktiviert 
dank seiner eigenen starken lexikalischen Prägung das Konzept Führer nicht, da es eindeutig mit dem Konzept Fahrerlaubnis assoziiert wird. Im dritten Beispiel dagegen gewährleistet der gesamte Satzkontext einen neutralen Charakter der Lesart und lässt dabei die Aktivierung des Konzepts Hitler verhindern.

Das oben genannte Lexem Führer fungiert jedoch primär als evaluative Einheit, die höchst emotional geprägt ist, und zwar sowohl in der Gegenwartssprache (negative Prägung) als auch in der damaligen NS-Sprache (positive Prägung). Nach Pechau's Ansicht bedarf die Steigerung der Bedeutung des Wortes Führer bis zur Verehrung keiner Erklärung - diese Feststellung sollte sich nicht nur auf das Lexem Führer selbst beziehen, sondern auch auf die daraus abgeleitete Anrede „Mein Führer“ (Pechau, 1937, S. 5 [1061]). Jeder zusätzliche Kommentar bezüglich des Wesens von derartigen Phrasen scheint dem Autor unnötig zu sein, da er ihn wie der Rest der damaligen Gesellschaft andächtig behandelt und die Tatsache einer solchen Erhöhung dieser mit Ehre ausgesprochenen Worte für ihn überaus offensichtlich sei. Doch selbst für den heutigen Empfänger zeigen diese Elemente in ihrer ganzen Pracht die damalige politische Wertschätzungskraft mit positiven Obertönen, sozialer Verehrung sowie voller Akzeptanz und bilden damit die Quintessenz des Nationalsozialismus.

Im Zusammenhang mit diesen Überlegungen ist nach Puzynina (2014, S. 10) anzumerken, dass uns einerseits egozentrisch, „eigennützig“ verstandene Werte wichtig sind, wir andererseits jedoch an solchen Werten interessiert sind, die für die ganze menschliche Gemeinschaft bedeutend sind. Eine tiefere Reflexion in dieser Hinsicht erfordert die Frage, inwieweit sowohl die ersteren als auch die letzten im Fokus unseres Interesses bleiben sollten. In der Ära des Nationalsozialismus war es eine Priorität, die für die ganze Gesellschaft relevanten nationalen Werte mit der offiziellen Interpretation der Wahrheit bzw. Nichtwahrheit zu verbinden. Das Reale, Wirkliche wurde als Wert an sich selbst wahrgenommen - positiv oder negativ in seiner Botschaft, aber den realen nationalen Bedürfnissen dieser und nicht einer anderen Interpretation entsprechend. Das, was nicht als wahr deklariert wurde, wurde eindeutig und ohne jede Möglichkeit der Opposition als Lüge proklamiert, die in den Augen der Nationalsozialisten direkt gegen die Nation - das Volk gerichtet war und eine starke Bedrohung für ihre Existenz darstelle. Ebenso entsteht das Problem der Interpretation, also bestimmten Phänomenen oder Situationen relevante und eindeutig korrekte Bedeutungen zu geben. Aus der Perspektive der emotional geprägten Sprache der Werte also evolvieren, wie 
Puzynina (2014, S. 8) schreibt, Probleme der Manipulation und der Lüge. Puzynina interpretiert diese beiden Phänomene als irreführend, fügt jedoch hinzu, dass sowie im Falle einer Manipulation es für einige beabsichtigte Profite des Manipulators führe, im Falle der Lüge dagegen müssen sie nicht obligatorisch auftauchen, weil es sogenannte „Notlügen“ („białe kłamstwa”) gebe. Im Kontext der politischen Angelegenheiten, die von den Ideologen des Nationalsozialismus geführt und propagiert wurden, kann man nach Puzynina weiter feststellen, dass bestimmten Einstellungen, Handlungen, Situationen, Ereignissen, Personen oder auch Institutionen durch Manipulation und Lüge üblicherweise positive bzw. negative Werte zugewiesen werden, die zwar der Realität nicht entsprechen, jedoch eine bestimmte politische Linie realisieren. Da also jede Lüge, Falschheit oder Unehrlichkeit die NS-Ideologie und dadurch auch das Volk direkt bedrohen sollten, musste dies eindeutig als negativer Wert verurteilt und stigmatisiert werden.

\section{6. „Neue Wörter“ als Indikatoren der sprachlich realisierten Bewertung}

„Neue Wörter" in der nationalsozialistischen Nomenklatur bilden vor allem solche Wörter, die zwar vorher formell bekannt waren, die jedoch in der „neudeutschen Sprache“ (Pechau, 1937, S. 2 [1058]) eine neue Bedeutung bzw. Auswirkungskraft erhielten. Obwohl Pechau selbst betont, dass der Sinn seiner Arbeit nicht darin bestehe, neue Wörter zu registrieren, sondern den Empfänger zum Nachdenken anzuregen, z. B. darüber, was diese Wörter zu vermitteln haben (ebd., S. 3 [1059]), scheint sein Beitrag diesen Auffassungen $\mathrm{zu}$ widersprechen. Der Rezipient braucht selbst über keine bestimmten verbalen Botschaften zu reflektieren, da der Autor dies selbst für ihn tut und dabei eine ausschließlich richtige Interpretation auferlegt. Um einen solchen Effekt zu erzielen, hat Pechau seinen Text mit Einheiten versorgt, die die Absichten des Autors explizit hervorheben und dabei keinen Widerspruch dulden. Er verwendet z. B. selbstverständlich, klar, deutlich, benutzt den Imperativ-Stil, missbraucht die Modalität durch ausgedrückte müssen-Sätze, Ausrufesätze oder erteilt im Text direkt die Anweisungen für korrekte Handlungsweisen:

(...) Deshalb ist selbstverständlich, wer nicht den richtigen Tritt des Volksmarsches hat, für den heißt es ,Tritt gewechselt'. Wer das Kommando nicht versteht, der muß nachexerzieren, bis er es gelernt hat. (Pechau, 1937, S. 2 [1058]) 
Unter dem Begriff, den er für diese „neuen Wörter“ verwendet, sind zahlreiche zitierte Angaben vermerkt sowie längere Phasen und schließlich einzelne Aussagen, die verschiedene Arten extremer Formulierungen enthalten (sowohl Invektiven als auch eindeutig nobilitierende Phrasen), die durch sprachliche Bewertungsmittel ausgedrückt wurden und die prinzipiell auf der lexikalischen Ebene im Text implementiert sind. Manchmal begleiten diese sprachlichen Einheiten entsprechende Kommentare bezüglich ihres semantischen Wertes oder einer bestimmten stilistischen Färbung. Daher verbirgt Pechau in der nationalsozialistischen Nomenklatur unter dem Begriff "neue Wörter" nicht nur Neologismen bzw. Okkasionalismen, die jedoch aufgrund von Einschränkungen in ihrem Verständnis ziemlich selten auftauchen, sondern auch alle Angaben im weiteren Sinne, die eine neue, nationalsozialistisch geprägte Botschaft sowie eine stark werdende Kraft aufweisen sollen. Zu den wichtigsten sprachlichen Bewertungsmitteln im Text gehören unter anderem lexikalische Einheiten, die positiv oder negativ geprägt sind (mit ihrer Färbung und Stärke abhängig von aktuell diskutierten ideologischen Fragen), Neologismen, Standardwörter (Fahnenwörter), Slogans (Schlagwörter), Epitheta, Metaphern oder Vergleiche. Unter den Textualitätskriterien fällt deutlich die Intentionalität auf, was darauf hinweist, dass der Autor der evolvierten nationalsozialistischen Ideologie folgt, die Intertextualität, da der Text sehr starke Verbindungen zu anderen primären Texten mit ähnlichen Propagandafunktionen aufweist ("Mein Kampf', "Der Mythus des 20. Jahrhunderts", gesammelte Reden von Goebbels), sowie die Situationalität, da die Gesamtheit solcher Faktoren, die den entstandenen Text für eine aktuelle bzw. rekonstruierbare Kommunikationssituation relevant machen, in der damaligen gesellschaftspolitischen Situation einen starken Einfluss auf den Empfänger ausüben sollte.

Eine der ebenfalls im diskutierten Text enthaltenen Bewertungsmethoden ist die (pseudo) Fachterminologie, die auch in „NichtFachtexten" missbraucht wurde. Sie sollte den Aussagen einen scheinbar wissenschaftlichen Charakter verleihen. Daher erscheinen im diskutierten Text neu entstandene Einheiten, meist im Bereich der politischen Rhetorik, wie: Novemberrevolte (6 [1062]), Futterkrippenpolitik (7 [1063]), Nadelstichpolitik, Gesinnungsakrobaten (8 [1064] ]) oder Tributzeppelin (9 [1065]), die gleichzeitig Anzeichen von phraseologisierten Einheiten aufweisen. Neben Zusammensetzungen (Komposita) erscheinen im Text als neue Wörter mit wertender Prägung auch zahlreiche Kollokationen aus dem Gebiet der politischen Rhetorik: preußischer Sozialismus (4 [1060]), parlamentarische 
Maulschafts (6 [1062]) sowie religiöse Referenzen: politische Methusalems (8 [1064]), koschere Anreizeutungsanstalt (9 [1065]), verorientalisches Christentum (14 [1070]). Bei der Bewertung der diskutierten Themen erfüllen diese Einheiten eine moralisierende und überzeugende Funktion im Text, die der Empfänger gemäß der Absicht des Emittenten entziffern soll (Kukowicz-Żarska, 2010, S. 137). Der Kontext der Verwendung einer bestimmten phraseologisierten Einheit kann sich auf ihre primäre Bedeutung beziehen, die für eine große Gruppe von Empfängern offensichtlich ist. Wenn dies jedoch nicht zutrifft, kann es dazu führen, dass die allgemeinen Wissensressourcen des Rezipienten erreicht werden (ebd., S. 136). Da die moralisierende und überzeugende Funktion dieser "neuen Wörter" in der NSNomenklatur eine primäre Rolle spielt, ist der Empfänger häufig dazu gezwungen, eben auf dieses Allgemeinwissen zurückzugreifen. Die wichtigste Aufgabe jeder bestimmten Einheit mit wertender Prägung besteht jedoch nicht darin, rein semantische bzw. etymologische Fragen zu erörtern. Der Schwerpunkt liegt hier vielmehr darauf, die endgültige Rezeption bestimmter Komposita oder Kollokationen im Kontext der Bewertung zu begreifen, damit sich der Empfänger hauptsächlich auf die projizierten Propagandainhalte konzentrieren kann.

\section{Militarisierung der Sprache im Hinblick auf die Bewertung}

Militarisierung der Sprache bzw. (pseudo)professionelle Terminologie im Bereich der Militärsprache sind im gesamten Text vorhanden. Die ersten Sätze der Veröffentlichung plädieren schon dafür:

Wir sprechen viel von politischer Ausrichtung des deutschen Menschen, auch von politischer Ausrichtung des wissenschaftlichen Arbeiters. Die Notwendigkeit dieser Ausrichtung wird auf vielen Gebieten aber ungern anerkannt. Und doch ist der gleiche Marschtritt die primitivste Voraussetzung für das Vorwärtskommen einer geschlossenen Truppe. Deshalb ist selbstverständlich, wer nicht den richtigen Tritt des Volksmarsches hat, für den heißt es 'Tritt gewechselt'. Wer das Kommando nicht versteht, der muß nachexerzieren, bis er es gelernt hat. Das ist einfache Tatsache in der Wissenschaft so selbstverständlich wie beim Militär. (Pechau, 1937, 2[1059])

Das aus der Militärsprache entnommene Verb ausrichten war sehr produktiv - im oben zitierten Abschnitt taucht es sogar dreimal als nominalisiertes Verb Ausrichtung auf - es zeichnete sich durch eine enorme Häufigkeit der Verwendung aus und war in den meisten schriftlichen Texten 
sowie Reden prominenter NS-Parteifunktionäre üblich. Dieses Lexem verwies auf eine unendliche Aufgabe, den Denk- und Gefühlsprozessen des ganzen Volkes eine völlig einheitliche nationalsozialistische Richtung $\mathrm{zu}$ geben (Schmitz-Berning, 1998). Es wurde mit dem Adjektiv politisch versorgt und bildete in der Nomenklatur des Nationalsozialismus den Begriff politische Ausrichtung. Dieser Pseudo-Fachterminus sollte den Anschein erwecken, dass bestimmte Texte und Äußerungen wissenschaftlich glaubwürdig sowie intellektuell verantwortlich sein mussten und dass sie sich sowohl auf das politische Denken eines "deutschen Bürgers" als auch auf eine politische Ausrichtung der "wissenschaftlichen Tätigkeit" beziehen sollten. Die Kollokation politische Ausrichtung sollte also als Fachbegriff für ein anerkanntes, politisches Konzept bzw. für einen gültigen politischen Standpunkt fungieren.

Im Kontext der emotionalisierten Sprache des Nationalsozialismus sind auch zahlreiche militärische Bezüge zu nennen, die hochgeschätzte, positiv konnotierte Eigenschaften widerspiegeln (z.B. Stärke, Mut, emotionale Distanz, Gelassenheit, Streben nach Machtgewinn, Disziplin) und die einen integralen Bestandteil davon bilden. Nur im oben zitierten Abschnitt finden wir eine ganze Reihe von militärischen Bezeichnungen: Militär, Truppe, Marschtritt, Volksmarsch, Kommando, Tritt - dies sind nur einige von zahlreichen ideologisierten Militär-Beispielen, die in den Texten von Ideologen dieser Zeit verwendet wurden. In Pechau's Beitrag gibt es sogar ein Kapitel unter dem Titel "Kampfsprachformen", in dem sich der Autor mit der Sprache befasst, die er selbst als Kampfsprache bezeichnet:

So mögen kurz die Worte genannt werden, die im Kampf des Nationalsozialismus gegen das ,System' geprägt worden sind. (...) Somit gestatten uns diese Wortformen, auch von dieser Seite her einen Einblick in den Kampf der Bewegung selbst zu gewinnen und ihre Markierung gewinnt dadurch historisch-wissenschaftliche Bedeutung. (Pechau, 1937, S. 5[1061])

Hier wird noch einmal die lexikalische Schicht erörtert, die Semantik von Wörtern bzw. Phrasen wurde jedoch zusätzlich im historischen sowie soziokulturellen Aspekt geschildert, was auch den vorgeplanten Bewertungszwecken dienen soll. Die meisten signifikanten Lexeme stammen aber nicht aus der Militärsprache, sondern aus der Sprache der politischen Debatte - der Autor versteht die Sprache des Kampfes als Kampf im Namen des Nationalsozialismus und nicht als Verwendung des Wortschatzes, der aus diesem Kampf selbst resultiert. 
Farbenbezeichnungen können auch eine produktive Wortbildungsbasis für Komposita sowie feste Wortbildungen aus dem Bereich der Militärsprache bilden. Zu diesen Farben gehört im Vordergrund das Adjektiv braun, das als Bezeichnung der Farbe von Uniformen in der NS-Zeit positiv wertende Konnotationen auslöste. Das Adjektiv braun fungiert für den Autor als produktives sprachliches Verfahren und gewährleistet die Bildung von folgenden Phrasen: die Braunhemden, braunes Heer, braunes Ehrenlied, braune Sturmkolonnen, Braunhemdenbeer (Pechau, 1937, S. 4f.[1060f.]). Alle Wortformationen, die auf der Basis des Braun-Lexems erstellt wurden, konnotierten eindeutig positiv, da sie von der Farbe der offiziellen SAUniformen (Sturmabteilungen der $N S D A P^{9}$ ) abgeleitet waren. Unter dem Einfluss der nationalsozialistischen Rhetorik wurde der primäre Begriff braune Hemden personifiziert und nicht mehr ausschließlich in der Bedeutung Objekt/Gegenstand (Teil der Uniform) verwendet - er evolvierte, wurde mit menschlichen Merkmalen versorgt und danach gewann eine zusätzliche signifikante und konnotative Neubedeutung Braunhemden - als Subjekt/Person (Mitglied der Sturmabteilungen der NSDAP).

\section{Schlussbemerkungen}

Evaluative Elemente in der NS-Sprache sowie die Wortkraft, die am Beispiel des Textes von Manfred Pechau geschildert wurde, bilden eine solide Grundlage für die Reflexion über die emotionsbezogene Sprache der damaligen Zeit. Die wertende Prägung der Äußerungen des Autors selbst sowie die Konnotationen, die sie bei den Emittenten hervorbringen, basieren auf der lexikalischen Ebene.

Die zitierten Phrasen beziehen sich größtenteils auf literarische, kulturelle bzw. historische Figuren und als solche erfüllen sie ihre übergeordnete Propaganda-Mission. Sprachstrategien, die die persuasive Handlung unterstützen, vermitteln eine positiv geprägte Mitteilung in Bezug auf die Anhänger des Nationalsozialismus und eine negative im Kontext jeglicher Opposition gegen die propagierten NS-Ideen. Laut Wodak (2008, S. 195f.) kann man diese Strategien als sich systematisch wiederholende Arten des Sprachgebrauchs charakterisieren, die mehr oder weniger detailliert definiert werden und bewusst als Kommunikationshandlungen übernommen werden, bei denen der Emittent versucht, mit Hilfe von entsprechend ausgewählten und explizite bzw. implizite geäußerten sprachlichen Mitteln

\footnotetext{
${ }^{9}$ Umgangssprachlich als Braunhemden genannt.
} 
seine eigenen Ziele - die sozialen, politischen oder psychologischen - zu erreichen. Dieses Verständnis der Kommunikationshandlungen kann man offensichtlich auf die emotionale und emotionalisierte Sprache des Nationalsozialismus beziehen.

\section{Bibliografie}

Fries, N. (2007). Die Kodierung von Emotionen in Texten. Teil 1: Grundlagen. JLT - Journal of Literary Theory, 2007/1(2), 293-337.

Fries, N. (2004). Gefühle, Emotionen, Angst, Furcht, Wut und Zorn. In W. Börner, K. Vogel (Hrsg.) Emotion und Kognition im Fremdsprachenunterricht (S. 3-24). Tübingen: Gunter Narr.

Jahr, S. (2000). Emotionen und Emotionsstrukturen in Sachtexten: Ein interdisziplinärer Ansatz zur qualitativen und quantitativen Beschreibung der Emotionalität von Texten. Berlin, New York: Walter de Gruyter.

Kämper-Jensen, H. Spracharbeit im Dienst des NS-Staats 1933 bis 1945. Zeitschrift für Germanistische Linguistik, 21/2, 150-183. https://doi.org/10.1515/zfgl.1993.21.2.150

Kukowicz-Żarska, K. (2018). O wartościowaniu i prawdzie w języku i tekście. Język narodowego socjalizmu w perspektywie językoznawczej i socjokulturowej. Forum Filologiczne Ateneum 1(6)2018, 61-86. https://doi.org/10.36575/23532912/1(6)2018.061

Kukowicz-Żarska, K. (2010). Funkcje związków frazeologicznych w tekstach doniesień prasy sportowej. Linguistica Bidgostiana, VII, 133-144. Bydgoszcz: Wydawnictwo Uniwersytetu Kazimierza Wielkiego.

Müller, S. (1994). Sprachwörterbücher im Nationalsozialismus. Die ideologische Beeinflussung von Duden, Sprach-Brockhaus und anderen Nachschlagewerken während des „Dritten Reichs“. Stuttgart: J.B. Metzler. https://doi.org/10.1007/ 978-3-476-04218-7

Lerchenmüller, J. \& Simon, G. (2009). Im Vorfeld des Massenmords. Germanistik und Nachbarfächer im 2. Weltkrieg. Eine Übersicht. Tübingen: GIFT Gesellschaft für interdisziplinäre Forschung Tübingen.

Pechau, M. (1937). Nationalsozialismus und deutsche Sprache. Nationalsozialistische Monatshefte. Zentrale politische und kulturelle Zeitschrift der NSDAP, 93, Dezember 1937, 2-16 [1058-1072]. München: Zentralverlag der NSDAP.

Pechau, M. (1935). Nationalsozialismus und deutsche Sprache. Diss. Greifswald. Greifswald: Hans Adler Buchdruckerei.

Puzynina, J. (2014). Kłopoty z nazwami wartości (i wartościami). Etnolingwistyka 26, 7-20. Lublin.

Puzynina, J. (1992). Język wartości. Warszawa: PWN. 
Schlosser, H.D. (2013). Sprache unterm Hakenkreuz. Eine andere Geschichte des Nationalsozialismus. Köln, Weimar, Wien: Böhlau. ISBN 978-3-412-21023-6

Sandig, B. (2007). Stilistische Funktionen von Phrasemen. In H. Burger et al. (Hrsg.) Phraseologie - Ein internationales Handbuch der zeitgenössischen Forschung. Band 1. (S. 158-174). Berlin, New York: Walter de Gruyter.

Schmidt, C. (2002). Wie wirken Wörter? Psycholinguistische Ansätze in der (feministischen) Sprachkritik. In J. Spitzmüller, K. S. Roth, B. Leweling, D. Frohning (Hrsg.) Streitfall Sprache. Sprachkritik als angewandte Linguistik? (S. 61-73). Bremen: Hempen.

Simon, G. (2003). NS-Sprache aus der Innensicht. Der Linguist Pechau und der Massenmord in den Sümpfen bei Loknja. In S. Ureland (Hrsg.) Convergence und Divergence of European Languages. (Studies in Eurolinguistics 1) (S. 277-303). Berlin: Logos. Abgerufen am 12 Oktober 2018 von: http://homepages.unituebingen.de/gerd.simon/pechau.pdf

Wodak, R. (2008). Dyskurs populistyczny: retoryka wykluczenia a gatunki języka pisanego. In: A. Duszak \& N. Fairclough (Hrsg.) Krytyczna Analiza Dyskursu. Interdyscyplinarne podejście do komunikacji społecznej (S. 185-213). Kraków: Towarzystwo Autorów i Wydawców Prac Naukowych UNIVERSITAS. 